\title{
Estudo anatômico das válvulas do tronco gastrocnêmio em cadáveres humanos
}

\author{
Anatomical study of valves in the gastrocnemius trunk \\ in human cadavers
}

\section{José Aderval Aragão1, Francisco Prado Reis², Luis Francisco Poli de Figueiredo ${ }^{3}$, Fausto Miranda Junior ${ }^{4}$, Guilherme Benjamin Brandão Pitta ${ }^{5}$}

\section{Resumo}

Contexto: As válvulas são estruturas características das veias, importantes na orientação do fluxo sangüíneo. Sua presença no sistema venoso superficial dos membros inferiores tem sido bastante estudada. No entanto, nas veias profundas, como a veia gastrocnêmia, a literatura é escassa.

Objetivo: Realizar um estudo anatômico das válvulas do tronco gastrocnêmio principal em cadáveres humanos adultos.

Métodos: Foram dissecados os troncos gastrocnêmios principais de 80 cabeças de músculos gastrocnêmios de 20 cadáveres adultos do sexo masculino, com idade entre 40 e 68 anos, após fixados e mantidos em solução de formol a $10 \%$. Os troncos e tipos de redes foram classificados de acordo com o proposto por Aragão et al. As válvulas foram estudadas quanto ao número, distribuição, localização e tipo com relação ao tronco, perna, cabeça do músculo e tipo de rede gastrocnêmia.

Resultado: Em 80 cabeças de músculos gastrocnêmios, foram encontrados 95 troncos gastrocnêmios principais, sendo que 17 deles eram duplicados. Foram encontradas 65 válvulas em 60 troncos gastrocnêmios principais, todas elas do tipo bicúspide, sendo 35 na rede tipo I, 23 na do tipo II e sete na rede tipo III. Em 74\% dos casos, as válvulas estavam localizadas no terço proximal do tronco gastrocnêmio principal.

Conclusão: As válvulas foram encontradas em todos os tipos de redes que possuíam tronco gastrocnêmio principal, eram todas do tipo bicúspide e se localizaram predominantemente no terço proximal dos troncos gastrocnêmios principais.

Palavras-chave: Insuficiência venosa, veias, anatomia.

\begin{abstract}
Background: Valves are characteristic structures of veins and are important to guide blood flow. Their presence in the superficial venous system of lower limbs has been well studied. However, there is a lack of published literature on deep veins, such as the gastrocnemius vein.

Objective: To carry out an anatomical study of the veins in the main gastrocnemius trunk in adult human cadavers.

Methods: The main gastrocnemius trunks of 80 gastrocnemius muscle heads of 20 adult cadavers were dissected. The cadavers were males, aged between 40 and 68 years, and fixed in 10\% formaldehyde solution. Trunks and net types were classified according to Aragão et al.'s proposal. The valves were studied as to quantity, distribution, location and type with regard to trunk, leg, muscle head and type of gastrocnemius net.
\end{abstract}

Results: Of 80 gastrocnemius muscle heads, there were 95 main gastrocnemius trunks, and 17 were duplicated. There were 65 valves in 60 gastrocnemius trunks, all of them bicuspid: 35 in type I net, 23 in type II and seven in type III net. Valves were located in the proximal third of the main gastrocnemius trunk in $74 \%$ of cases.

Conclusion: Valves were found in all net types that had main gastrocnemius trunk. These valves were bicuspid and predominantly located in the proximal third of main gastrocnemius trunks.

Keywords: Venous insufficiency, veins, anatomy.

1. Professor assistente, Universidade Federal de Sergipe (UFS), Aracaju, SE. Professor adjunto III, Universidade Tiradentes (UNIT), Aracaju, SE. Doutorando, Curso de Pós-Graduação em Angiologia e Cirurgia Vascular, Universidade Federal de São Paulo (UNIFESP), São Paulo, SP.

2. Professor titular, UNIT, Aracaju, SE. Coordenador, Laboratório de Morfologia e Biologia Estrutural, Instituto de Tecnologia e Pesquisa (ITP), UNIT, Aracaju, SE.

3. Professor titular, Departamento de Cirurgia, UNIFESP, São Paulo, SP.

4. Professor titular, UNIFESP, São Paulo, SP.

5. Professor adjunto, Universidade Estadual de Ciências da Saúde de Alagoas (UNCISAL), Maceió, AL.

Artigo submetido em 05.03.07, aceito em 27.04.07. 


\section{Introdução}

A presença de válvulas é uma característica das veias $^{1}$. Por sua base, as válvulas estão implantadas na parede venosa, possuem na sua estrutura elementos fibrosos, são mais numerosas em fetos, onde algumas vezes aparecem com aspecto de incompletas, e tendem a desaparecer nos adultos ${ }^{2}$. As válvulas são mais freqüentes nas veias dos membros inferiores, onde dirigem o fluxo, impedindo também o refluxo sangüíneo ${ }^{2,3}$. Os primeiros estudos a respeito das válvulas remontam a 1854 e têm sido atribuídos a Houlé ${ }^{4}$.

É reconhecida a importância da influência valvular na patogenia das varizes, e isso tem levado vários autores a estudarem a anatomia das válvulas nos membros inferiores ${ }^{4-9}$. Para a maioria desses autores, as veias musculares possuem válvulas, com possível exceção das veias soleares e gastrocnêmias. Thiery ${ }^{10}$ atribuiu à destruição valvular a base da inversão da corrente sangüínea das veias profundas para as superficiais. Hobbs ${ }^{11}$ destacou que as veias gastrocnêmias dilatadas por insuficiência venosa eram flebograficamente visíveis.

De acordo com Browse $^{3}$, a grande variação do número de válvulas nas veias musculares da panturrilha tem dificultado, ou impedido, até o presente, de estabelecer sua sistematização. Poucos autores têm feito referência à presença de válvulas nas veias gastrocnêmias $^{3,12-15}$. Verberck ${ }^{16}$ e Stritecky-Kahlek ${ }^{17}$ afirmaram que existia uma válvula na parte terminal da veia gastrocnêmia. Tretbar ${ }^{18}$ descreveu que as veias gastrocnêmias possuem mais válvulas do que as veias soleares. Villallonga ${ }^{19}$ destacou a importância das válvulas gastrocnêmias na orientação do fluxo sangüíneo durante os movimentos de contração e relaxamento muscular. Marques $^{20}$ afirmou que as veias gastrocnêmias não possuem válvulas.

Embora a maioria dos autores destaque o papel morfofuncional desempenhado pelas válvulas venosas, verifica-se que é escassa ou inexistente uma descrição sistemática das válvulas, tanto nas veias gastrocnêmias como no tronco gastrocnêmio principal. Por essa razão, foi realizado o presente estudo, que procurou descrever a anatomia das válvulas do tronco gastrocnêmio principal observando os aspectos: número, tipo, distribuição e localização.

\section{Método}

Foram utilizados 40 membros inferiores de 20 cadáveres humanos adultos, todos do sexo masculino, com idade entre 40 e 68 anos, fixados e mantidos havia mais de um ano em solução de formol a $10 \%$. Os cadáveres pertenciam aos laboratórios de anatomia das Universidades Federal de Sergipe, Tiradentes, Federal da Bahia e Estadual de Ciências da Saúde de Alagoas. O material foi usado de acordo com a Lei 8501, de 30 novembro de 1992, que dispõe sobre a utilização de cadáveres não reclamados para fins de estudo ou pesquisa científica. $\mathrm{O}$ projeto foi aprovado pelos comitês de ética em pesquisa da Universidade Estadual de Ciências da Saúde de Alagoas (UNCISAL), protocolo número 038/02 e Universidade Federal de São Paulo (UNIFESP), protocolo número 1.475/03.

A presença, o número, a distribuição, a localização e o tipo de válvula do tronco gastrocnêmio foram estudados com relação à perna, à cabeça do músculo gastrocnêmio e ao tipo de rede gastrocnêmia. $\mathrm{O}$ tronco gastrocnêmio principal e tipos de rede foram classificados de acordo com o proposto por Aragão et al. ${ }^{21}$ Esses autores classificaram quatro diferentes tipos de redes, onde todo o sistema de veias gastrocnêmias drenava diretamente ou através de troncos colaterais e axiais para o tronco venoso gastrocnêmio principal. Os dados resultantes das dissecações anatômicas foram documentados através de fotografia digital e tabelas.

\section{Resultados}

Nas 80 cabeças dos 40 músculos gastrocnêmios estudados, foram encontrados 95 troncos gastrocnêmios principais distribuídos por perna e cabeça de músculo. Desses troncos, 61 eram únicos e 17 duplicados (Tabela 1). Foram encontradas também 65 válvulas distribuídas em número aproximadamente igual em ambas as pernas (Tabela 2). Todas as válvulas encontradas eram do tipo bicúspide (Figura 1). 
Quanto à localização das válvulas, elas foram predominantes no segmento proximal, em aproximadamente $74 \%$ dos casos, e ocorreram em maior número na cabeça medial da perna esquerda. No segmento médio, ocorreram cerca de $17 \%$ dos casos, e no segmento distal apenas $9 \%$. Neste último segmento, merece destaque o fato de

Tabela 1 - Troncos gastrocnêmios principais: distribuição por perna e cabeça de músculo

\begin{tabular}{lcc}
\hline & \multicolumn{2}{c}{ Número de troncos } \\
\cline { 2 - 3 } Perna/Cabeça de músculo & Únicos & Duplicados \\
\hline Direita & 13 & 07 \\
Medial & 16 & 04 \\
Lateral & 29 & 11 \\
Subtotal & & \\
Esquerda & 14 & 05 \\
Medial & 18 & 01 \\
Lateral & 32 & 06 \\
Subtotal & 61 & 17 \\
Total &
\end{tabular}

todas as cabeças do músculo gastrocnêmio possuem o mesmo número de válvulas, exceção feita para a cabeça medial da perna esquerda, onde ocorreu a presença de mais de uma válvula no tronco gastrocnêmio (Tabela 3).

A distribuição das válvulas nos troncos gastrocnêmios principais, considerando o tipo de rede e cabeça de músculo gastrocnêmio (Tabela 4), mostrou 35 válvulas na rede tipo I, 23 na rede tipo II e sete na rede tipo III (Tabela 5). O número de troncos sem válvulas nas redes do tipo I e II foi semelhante, enquanto na rede tipo III ocorreram sete troncos sem válvulas. Considerando-se os troncos sem válvulas e os troncos duplicados, o número de troncos com válvulas por tipo de rede teve a seguinte distribuição: 31 na rede tipo I, 22 na rede tipo II e 7 na do tipo III.

Foram encontradas válvulas em todos os tipos de rede. Quanto a sua distribuição por tipo de rede, perna e cabeça de músculo gastrocnêmio, o maior número de válvulas foi encontrado nas pernas e cabeça de músculo da rede tipo I. Isoladamente, ocorreu um maior número de válvulas na rede do tipo I na perna direita. O número de válvulas foi sempre maior nas cabeças laterais, com

Tabela 2 - Distribuição das válvulas: distribuição de tronco por perna e por cabeça do músculo gastrocnêmio

\begin{tabular}{ccccc}
\hline & & \multicolumn{3}{c}{ Número de válvulas por tronco } \\
\cline { 3 - 5 } $\begin{array}{l}\text { Perna/Cabeça } \\
\text { do músculo }\end{array}$ & $\begin{array}{c}\text { Número } \\
\text { de troncos }\end{array}$ & $\mathbf{0}$ & $\mathbf{1}$ & $\mathbf{2}$ \\
\hline Direita & & & 13 & 01 \\
Medial & 27 & 08 & 13 & 01 \\
Lateral & 24 & 21 & 28 & 02 \\
Subtotal & 51 & & & \\
Esquerda & & 07 & 15 & 02 \\
Medial & 24 & 07 & 12 & 01 \\
Lateral & 20 & 14 & 27 & 05 \\
Subtotal & 44 & 35 & 55 & \\
Total & 95 & & &
\end{tabular}


exceção nos troncos gastrocnêmios das cabeças mediais das pernas esquerda e direita da rede tipo II (Tabela 6).

Tabela 3 - Topografia das válvulas no tronco gastrocnêmio: distribuição por perna e cabeça

\begin{tabular}{ccc}
\hline $\begin{array}{l}\text { Perna/Cabeça } \\
\text { de músculo }\end{array}$ & $\begin{array}{c}\text { Segmento } \\
\text { do tronco }\end{array}$ & $\begin{array}{c}\text { Número } \\
\text { de válvulas }\end{array}$ \\
\hline Direito & Proximal & \\
Medial & Médio & 12 \\
& Distal & 02 \\
Lateral & Proximal & 01 \\
& Médio & 12 \\
Subtotal & Distal & 04 \\
Esquerdo & & 01 \\
Medial & & 32 \\
& Proximal & 12 \\
& Médio & 04 \\
Lateral & Distal & 03 \\
& Proximal & 12 \\
Subtotal & Médio & 01 \\
Total & Distal & 01 \\
& & 33 \\
& & 65 \\
\hline
\end{tabular}

Tabela 4 - Troncos gastrocnêmios principais por tipo de rede e cabeça de músculo

\begin{tabular}{llc}
\hline & \multicolumn{2}{c}{ Número de troncos por perna } \\
\cline { 2 - 3 } Tipo de rede/ & Direita & Esquerda \\
Cabeça de músculo & & \\
\hline I $\quad$ Medial & 11 & 11 \\
Lateral & 13 & 09 \\
II $\quad$ & 11 \\
$\quad$ Medial & 07 & 09 \\
Lateral & & \\
III & 05 & 03 \\
Medial & 04 & 02 \\
Lateral & 51 & 44 \\
Total &
\end{tabular}

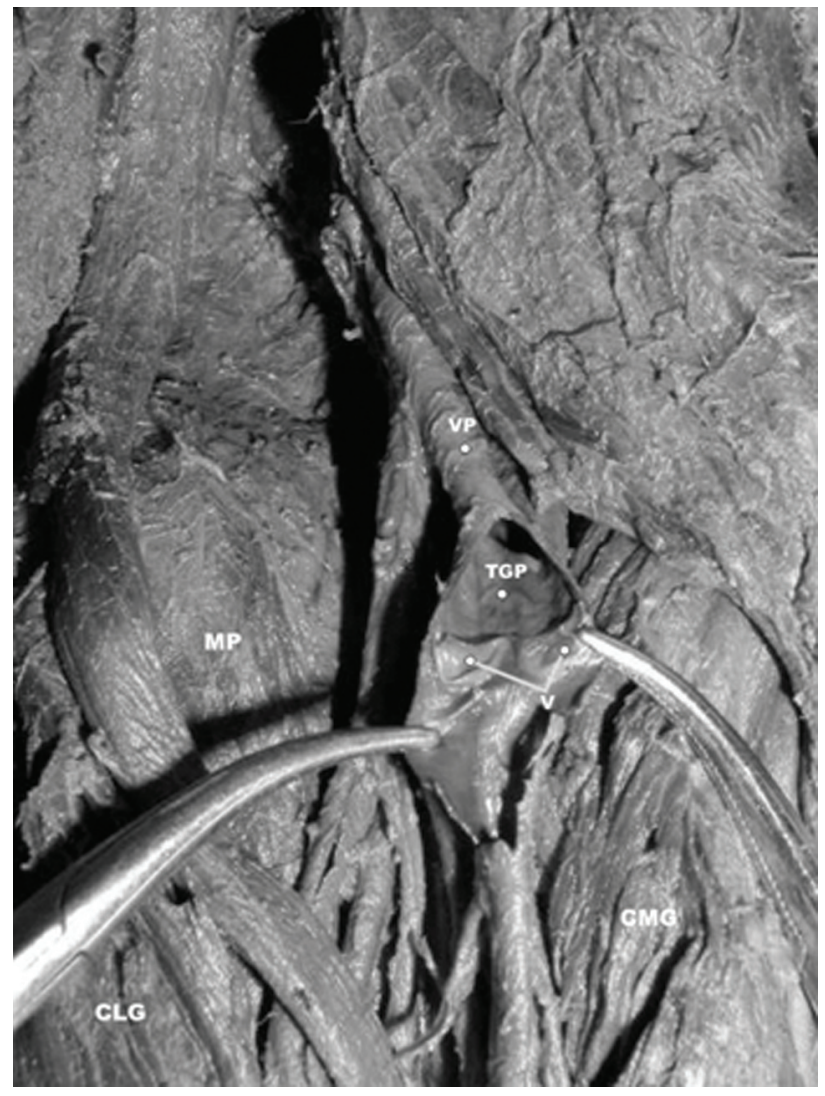

CLG = cabeça lateral do músculo gastrocnêmio; $\mathrm{CMG}=$ Cabeça medial do músculo gastrocnêmio; $\mathrm{MP}=$ Músculo poplíteo; TGP = Tronco gastrocnêmio principal; $\mathrm{V}=$ Válvula; $\mathrm{VP}=$ Veia poplítea.

Figura 1 - Válvula do tipo bicúspide no tronco gastrocnêmio principal

Das 35 válvulas encontradas na rede tipo I, a maioria estava localizada no segmento proximal do tronco gastrocnêmio principal. Apenas no segmento médio do tronco da cabeça lateral do músculo gastrocnêmio da perna esquerda não ocorreu a presença de

Tabela 5 - Válvulas e suas ausências por tipo de rede

\begin{tabular}{lcc}
\hline Tipo de rede & Número de válvulas & Ausência de válvulas \\
\hline I & 35 & 13 \\
II & 23 & 15 \\
III & 07 & 07 \\
Total & 65 & 35 \\
\hline
\end{tabular}


Tabela 6 - Válvulas no tronco gastrocnêmio principal por tipo de rede, perna e cabeça de músculo

\begin{tabular}{lcc}
\hline \multicolumn{1}{c}{ Tipo de rede/Perna } & Cabeça de músculo & Número de válvulas \\
\hline I & Medial & \\
Direita & Lateral & 09 \\
& Medial & 10 \\
Esquerda & Lateral & 09 \\
& & 07 \\
II & Medial & \\
Direita & Lateral & 04 \\
\multirow{2}{*}{ Esquerda } & Medial & 06 \\
& Lateral & 08 \\
III & & 05 \\
Direita & Medial & \\
& Lateral & 02 \\
Esquerda & Medial & 01 \\
Total & Lateral & 02 \\
\hline
\end{tabular}

válvula. Na rede tipo II, suas 23 válvulas foram predominantemente localizadas no segmento proximal dos troncos. No segmento distal, ocorreram válvulas apenas na cabeça medial esquerda do músculo gastrocnêmio. As sete válvulas presentes na rede tipo III estavam localizadas no segmento proximal de todos os troncos gastrocnêmios principais (Tabela 7).

\section{Discussão}

Nosso estudo mostrou que, de 95 troncos gastrocnêmios principais, foram encontradas 65 válvulas em 60 troncos. Autores como Vandendriessche ${ }^{12}$, Schinder, Ramelet $^{13}$, Mello $^{14}$, Hobbs $^{15}$ e Tretbar $^{18}$ admitiram a presença de válvulas na veia gastrocnêmia. Para Verberck ${ }^{16}$ e Stritecky-Kahler ${ }^{17}$ existiria uma válvula na parte terminal da veia gastrocnêmia. A maioria desses autores, entretanto, não quantificou nem estabeleceu a topografia dessas válvulas tanto nas veias como no tronco gastrocnêmio principal.
Foram encontradas válvulas em 70,4\% dos troncos gastrocnêmios principais da rede tipo I, em 59,0\% dos troncos da rede tipo II, e em 50\% dos troncos da rede tipo III. Quanto à relação entre o número de válvulas por rede e tronco gastrocnêmio principal, 79,5\% ocorreu na rede tipo I; $62,1 \%$ na rede tipo II e $50 \%$ na rede tipo III. As redes tipo I e II tiveram quase o mesmo número de troncos sem válvulas. A rede do tipo III teve igual número de troncos com e sem válvula. A proposta original de Aragão et al. ${ }^{21}$ a respeito das redes venosas gastrocnêmias e a classificação de suas veias nos permitiu observar as características anatômicas da distribuição das válvulas nos troncos gastrocnêmios de acordo com o tipo de rede. Entretanto, achados semelhantes na literatura compulsada, parecem escassos ou inexistentes.

De acordo com Aragão ${ }^{22,23}$, das 438 veias gastrocnêmias dissecadas, 231 pertenciam à rede tipo II, 174 à rede tipo I e 28 à rede tipo III. Esses dados nos 
Tabela 7 - Topografia das válvulas no tronco gastrocnêmio principal

\begin{tabular}{|c|c|c|c|}
\hline Tipo de rede/Perna & Cabeça de músculo & Segmento & Número de válvulas \\
\hline \multicolumn{4}{|l|}{ I } \\
\hline \multirow[t]{6}{*}{ Direita } & Medial & Proximal & 07 \\
\hline & & Médio & 01 \\
\hline & & Distal & 01 \\
\hline & Lateral & Proximal & 07 \\
\hline & & Médio & 02 \\
\hline & & Distal & 01 \\
\hline \multirow[t]{6}{*}{ Esquerda } & Medial & Proximal & 05 \\
\hline & & Médio & 02 \\
\hline & & Distal & 02 \\
\hline & Lateral & Proximal & 06 \\
\hline & & Médio & - \\
\hline & & Distal & 01 \\
\hline \multicolumn{4}{|l|}{ II } \\
\hline \multirow[t]{6}{*}{ Direita } & Medial & Proximal & 03 \\
\hline & & Médio & 01 \\
\hline & & Distal & - \\
\hline & Lateral & Proximal & 04 \\
\hline & & Médio & 02 \\
\hline & & Distal & - \\
\hline \multirow[t]{6}{*}{ Esquerda } & Medial & Proximal & 05 \\
\hline & & Médio & 02 \\
\hline & & Distal & 01 \\
\hline & Lateral & Proximal & 04 \\
\hline & & Médio & 01 \\
\hline & & Distal & - \\
\hline \multicolumn{4}{|l|}{ III } \\
\hline \multirow[t]{6}{*}{ Direita } & Medial & Proximal & 02 \\
\hline & & Médio & - \\
\hline & & Distal & - \\
\hline & Lateral & Proximal & 01 \\
\hline & & Médio & - \\
\hline & & Distal & - \\
\hline \multirow[t]{6}{*}{ Esquerda } & Medial & Proximal & 02 \\
\hline & & Médio & - \\
\hline & & Distal & - \\
\hline & Lateral & Proximal & 02 \\
\hline & & Médio & - \\
\hline & & Distal & - \\
\hline Total & & & 65 \\
\hline
\end{tabular}


revelaram que o número de válvulas não foi diretamente relacionado com o número de veias da rede.

Em vista da escassez de estudos semelhantes, cremos que os nossos achados anatômicos representam uma contribuição de interesse para ajudar na compreensão da complexa drenagem venosa dos membros inferiores, em especial, a do sistema sóleo-gemelar. É difícil estabelecer com este tipo de estudo anatômico o papel dos nossos achados na fisiologia e fisiopatologia. Entretanto, acreditamos que, no futuro, eles possam servir como base para estudos hemodinâmicos, através flebografia, ultra-sonografia e pletismografia. Isso poderá ainda ajudar cirurgiões vasculares e imagenologistas na interpretação de seus achados.

\section{Conclusão}

O número de válvulas presentes nos troncos gastrocnêmios principais variou em relação ao tipo de rede gastrocnêmia, cabeça do músculo gastrocnêmio e perna. As válvulas ocorreram em todos os tipos de rede, foram localizadas predominantemente no terço proximal do tronco gastrocnêmio principal e todas eram do tipo bicúspide.

\section{Referências}

1. Di Dio LJA. Sistema cardiovascular. In: Di Dio LJA. Tratado de anatomia sistêmica aplicada. $2^{\text {a }}$ ed. São Paulo: Atheneu; 2002. p. 299-430.

2. Garrido MBM. Anatomia médico-cirúrgica do sistema venoso dos membros inferiores. In: Maffei FHA. Doenças vasculares periféricas. $3^{\mathrm{a}}$ ed. Rio de Janeiro: Medsi; 2002. Vol I. p. $133-68$.

3. Browse NL, Burnand KG, Irvine AT, Wilson NM. Insuficiência venosa crônica. In: Browse NL, Burnand $\mathrm{KG}$, Irvine AT, Wilson NM. Doenças venosas. $2^{\text {a }}$ edição. Rio de Janeiro: DiLivros; 2001. p. 353-74.

4. Bouchet A. Anatomie morphologique des valvules des membres inférieurs. Phlebologie. 1992;45:233-44; discussion 244-5.

5. Kwakye LB. Frequency and distribution of the valves in the large deep and superficial veins of the lower limb in the aged. Acta Morphol. Neerl Scand. 1971;9:41-6.

6. Vasconcelos, LAR. Estudo anatômico das válvulas do segmento fêmoro-popliteo em cadáveres humanos adultos [dissertação]. São Paulo: Universidade Federal de São Paulo; 2006.

7. Van Cleef JF, Hugentobler JP, Desvaux P, Griton P, Cloarec M. Quelques aspects endoscopiques de valvules chez le variqueux. Phlebologie. 1991;44:623-7.
8. Gillot C, Gillot JM. Variations et valvulation du système tronculaire fémoro-poplité. Phlebologie. 1991;44:537-74; discussion 575-6.

9. Blanchemaison P. Intérêt de l'endoscopie veineuse dans l'exploration et le traitement de l'insuffisance veineuse des membres inférieurs. J Mal Vasc. 1992;17 Suppl B:109-12.

10. Thiery L. Etiopatologia de las dilataciones venosas. Angiologia. 1975;27:272-8.

11. Hobbs JT. Errors in the differential diagnosis of incompetence of the popliteal vein and short saphenous vein by Doppler ultrasound. J Cardiovasc Surg (Torino). 1986;27:169-74.

12. Vandendriessche M. Le rapport entre l'insuffisance des veines. jumelles et les varices. Phlebology. 1989;42:171-84.

13. Schnider P, Ramelet AA. Anatomia. In: Ramelet AA, Monti M. Manual de flebologia. Barcelona: Masson; 1992. p. 3-19.

14. Mello NA. Grandes sindromes venosas. In: Mello NA. Angiologia. Rio de Janeiro: Guanabara Koogan; 1998. p. 253-9.

15. Hobbs JT. The enigma of the gastrocnemius vein. Phlebology. 1988;3:19-30.

16. Verberch AW. Sistema venoso soleo-gemelar: su rol en la insuficiencia venosa de miembros [tese]. Córdoba: Universidad Nacional de Córdoba; 1997.

17. Stritecky-Kähler T. Anatomia del sistema venoso. In: Stritecky-Kähler T. Cirugía de las venas varicosas. Madrid: Marban Libros; 1997. p. 2-17.

18. Tretbar LL. Deep veins. Dermatol Surg. 1995;21:47-51.

19. Vilallonga JL, Ulloa J, Manresa EV. Microcirculacion venosa. In: $6^{\circ}$ Congresso Panamericano de Flebologia e Linfologia. Memorias del Symposium zyma sobre microcirculacion; 1994; Cartagena. Anais. Cartagena: Sociedad Panamericana de Flebologia e Linfologia; 1994. p. 73-92.

20. Marques JS. [Varices caused by twin vein insufficiency. Value of phlebography]. Phlebologie. 1972;25:113-23.

21. Aragao JA, Reis FP, Pitta GB, Miranda F Jr., Poli de Figueiredo LF. Anatomical study of the gastrocnemius venous network and proposal for a classification of the veins. Eur J Vasc Endovasc Surg. 2006 Apr;31(4):439-42. Epub 2005 Dec 15.

22. Aragão JA. Estudo anatômico das veias gastrocnêmias em cadáveres humanos adultos [dissertação]. São Paulo: Universidade Federal de São Paulo; 2004.

23. Aragão JA, Reis FP, Figueiredo LF de P, Pitta GBB, Miranda Jr. F. Anatomia das veias e troncos gastrocnêmios em cadáveres humanos adultos. J Vasc Bras. 2004;3:297-303.

Correspondência:

José Aderval Aragão

Rua Aloísio Campos, 500 - Atalaia

CEP 49035-020 - Aracaju, SE

Tel.: (79) 3255.1381, (79) 9989.6767

E-mail: jaafelipe@infonet.com.br 